\title{
Meningitis Secondary to Pseudomonal Endophthalmitis in a Burn Patient: A Case Report and Review of the Literature
}

\author{
Sergio E. Perez and Linwood R. Haith
}

\begin{abstract}
Background: To report the case of a burn patient with meningitis secondary to pseudomonal endophthalmitis that led to death.

Case Presentation: An 18-year-old female who sustained flame burns in a house fire was admitted to our burn center. She sustained burns that accounted for $68 \%$ of her total body surface area, including her face. The patient underwent multiple excision and grafting procedures. On the thirteenth post-burn day, wound cultures revealed a resistant Pseudomonas sensitive only to aminogyclosides and colistin. On the twentieth post-burn day the patient developed edema, erythema, and proptosis of the right eye. Ophthalmology was consulted and endophthalmitis was diagnosed. A lateral right canthotomy was performed. Treatment modalities including intra-vitreous antibiotic injection were discussed but not performed. Computed tomography (CT) of the head at that time showed concern for retrobulbar inflammation. There was a progressive decrease in her mental status. An additional brain CT scan showed diffuse cerebral abnormalities and edema. Clinical examination at that time demonstrated decreased cerebral activity consistent with brain death and repeat neurologic examination confirmed brain death. Conclusion: Meningitis constitutes a rare complication of endophthalmitis and its prompt diagnosis and treatment via administration of systemic antibiotics via intravenous or intrathecal routes, with intra-vitreal antibiotics could lead to a more favorable outcome.
\end{abstract}

Keywords: burn infection; facial burns; intra-vitreal antibiotics; multi-drug resistant pseudomonas; pseudomonas endophthalmitis; pseudomonas meningitis

$\mathbf{E}$ NDOPHTHALMITIS is a rare condition, usually occurring as a complication of intra-ocular surgery or penetrating injury. Rarely, endophthalmitis may follow metastatic spread of fungal or bacterial infection from a distant source and the majority of these patients suffer from an underlying disease. Pre-disposing factors are immunocompromise, intravenous drug abuse, and prolonged stay in intensive care.

\section{Case Presentation}

An 18-year old female of Caucasian origin was admitted to the Nathan Speare Regional Burn Treatment Center
(Upland, PA) with $68 \%$ total body surface area flame burns sustained in a house fire. The body areas of involvement included the patient's face, torso, and upper and lower extremities. The patient also sustained a severe upper airway injury discovered on bronchoscopy. The patient underwent multiple excision and grafting procedures on the second, fourth, and tenth post-burn days. On the eleventh post-burn day, the patient underwent excision and temporary closure of facial wounds. Additional excision and grafting procedures were performed on the seventeenth and nineteenth post-burn days. Early nutritional supplementation with tube feedings was commenced and advanced to goal as tolerated.

Nathan Speare Regional Burn Treatment Center, Crozer-Chester Medical Center, Upland, Pennsylvania.

(C) Sergio E. Perez and Linwood R. Haith 2017; Published by Mary Ann Liebert, Inc. This Open Access article is distributed under the terms of the Creative Commons License (http://creativecommons.org/licenses/by/4.0), which permits unrestricted use, distribution, and reproduction in any medium, provided the original work is properly credited. 
On the thirteenth post-burn day, wound cultures obtained from the right peri-orbital wound revealed a multi-drugresistant Pseudomonas that was sensitive only to aminogyclosides and colistin. The patient was started on intravenous gentamicin. On the twentieth post-burn day, the patient developed edema, erythema, and proptosis of the right eye for which an ophthalmologic evaluation was obtained. Intra-ocular pressure was elevated and CT scan of the head showed retrobulbar inflammation. The diagnosis of endophthalmitis was made. A right lateral canthotomy was performed. Topical ophthalmic gentamicin drops were initiated. Vitreal tap and intra-vitreal antibiotic injection were discussed but not performed. In the days after this diagnosis, there was progressive decrease in the patient's mental status. On the day before death, the patient was noted to have left ocular nystagmus. A CT scan of the brain showed diffuse cerebral abnormalities and edema. Clinical examination at that time showed decreased cerebral activity consistent with brain death and repeat neurologic examination confirmed brain death. Post mortem examination revealed meningitis as the cause of death with accompanying endophthalmitis.

\section{Discussion and Literature Review}

Endophthalmitis is a rare serious infection of the interior eye. It occurs from micro-organisms gaining entry into the eye after surgery, penetrating trauma, or a distant source of infection somewhere else within the body. This latter mode of entry is also known as endogenous or metastatic endophthalmitis. Endogenous endophthalmitis is rare and accounts for only a small percentage of reported cases $(2 \%-$ $8 \%$ ) [1]. Fungal micro-organisms are responsible for the majority of endogenous cases [2]. Gram-negative microorganisms cause approximately one-third of all endogenous cases. In the majority of reported cases of endogenous endophthalmitis, these patients usually have an underlying medical condition that is associated with immunocompromise. In one study, $56 \%$ of patients with endogenous endophthalmitis had an immunocompromising condition, many of whom had type 2 diabetes mellitus [3]. Intravenous drug abuse, prolonged intensive care unit stay, and immunosuppressive treatment have also been associated with cases of endogenous endophthalmitis [3]. It is well known that burn patients suffer post-burn immunosupression, which was most likely a major contributing factor in our patient's case. Only two previous case reports of endophthalmitis associated with burns have been reported $[4,5]$. In addition, there have only been two previous case reports of meningitis following endophthalmitis, both of which occurred post-operatively in patients who underwent ocular surgery $[6,7]$.

Once diagnosed, treatment with broad-spectrum antibiotics should be instituted. Currently, the broad-spectrum antibiotic regimen recommended for endophthalmitis is intravitreal vancomycin and ceftazidine [8]. Vitreous cultures may be obtained to identify the causative microorganism although only approximately $74 \%$ of vitreous cultures are positive in cases of endogenous bacterial endophthalmitis [9]. In this patient's case, it can be assumed that the causative organism was the multi-drug-resistant Pseudomonas and intra-vitreal gentamicin may have been of benefit. Intra-vitreal gentamicin has been reported to be more toxic compared with ceftazidine because of the potential for macular infarction [10]. However, in retrospect and given the extensive multi-drug-resistance of this organism, the potential benefit would have outweighed the overall outcome. In severe cases of endophthalmitis that are non-responsive to antibiotic therapy, a vitrectomy is indicated. Because of the rapid disease progression, the patient's critical condition, lack of experience and resources by our ophthalmology department in treating endogenous bacterial endophthalmitis, and lack of suspicion for an infectious etiology causing her mental status changes, this ultimately led to the patient's death.

\section{Conclusion}

Endogenous endophthalmitis is rare in the general population and exceedingly rare in burn patients. A literature search revealed only two prior cases. Despite its rarity, it is a serious and potentially deadly disease process. In this case it appears that the endophthalmitis was precipitated by the antibiotic-resistant pseudomonal infection that led to meningitis and ultimately death. Burn surgeons should be vigilant in cases involving burns of the face when resistant Pseudomonas is prevalent and should be aware of the possible complications. Early recognition and immediate treatment are necessary to prevent further progression of the disease process and achieve a reasonable outcome.

\section{Author Disclosure Statement}

No competing financial interests exist.

\section{References}

1. Lemley CA, Han DP. Endophthalmitis: A review of current evaluation and management. Retina 2007;27:662-680.

2. Romero CF, Rai MK, Lowder CY, Adal KA. Endogenous endophthalmitis: Case report and brief review. Am Fam Physician 1999;60:510-514.

3. Jackson TL, Eykyn SJ, Graham EM, Stanford MR. Endogenous bacterial endophthalmitis: A 17-year prospective series and review of 267 reported cases. Surv Ophthalmol 2003;48:403-423.

4. Snow, DG, Eagling EM, Lawrence JC. Bilateral metastatic endophthalmitis as a complication of major burns. Burns 1992;18:342-344.

5. Jain ML, Garg AK. Metastatic endophthalmitis in a patient with major burns: A rare complication. Burns 1995;21: 72-73.

6. Ali K, Owen M, Kumar I, Cazabon S. Streptococcus pneumoniae meningitis following postoperative endophthalmitis. Eye 2012;26:1593.

7. Chan, SM, Hodge WG, Leonard BC. Postoperative Streptococcus pneumoniae endophthalmitis complicated by meningitis. Arch Opthalmol 1998;116:951-953.

8. Bertino JS. Impact of antibiotic resistance in the management of ocular infections: The role of current and future antibiotics. Clin Ophthalmol 2009;3:507-521.

9. Okada AA, Johnson RP, Liles WC, et al. Endogenous bacterial endophthalmitis: Report of a 10-year retrospective study. Ophthalmology 1994;101:832-838. 
10. Campochiaro PA, Lim JI. Aminoglycoside toxicity in the treatment of endophthalmitis. Arch Ophthalmol 1994;112: $48-53$.

Address correspondence to: Dr. Sergio E. Perez Nathan Speare Regional Burn Treatment Center Crozer-Chester Medical Center One Medical Center Boulevard, Suite 241 Upland, PA 19013

E-mail: sergiope@pcom.edu

\section{Abbreviation Used}

$\mathrm{CT}=$ computed tomography
Cite this article as: Perez SE and Haith LR (2017)

Meningitis secondary to pseudomonal endophthalmitis in a burn patient: A case report and review of the literature. Surgical Infections Case Reports 2:1, 95-97, DOI: 10.1089/crsi.2016.0026 